\title{
Multi-Objective Compliance Control of Redundant Manipulators: Hierarchy, Control, and Stability
}

\author{
Alexander Dietrich, Christian Ott, and Alin Albu-Schäffer \\ Institute of Robotics and Mechatronics, DLR. Contact: Alexander.Dietrich@dlr.de
}

\begin{abstract}
Robots with a large number of actuated degrees of freedom are usually redundant w. r.t. a given task. That kinematic redundancy can be utilized to execute additional tasks simultaneously, e.g. via null space projection techniques. We introduce a new representation of hierarchical robot dynamics which are based on a set of particular null space velocities. Dynamic consistency is preserved, and strict compliance with the order of priority is ensured at all times due to a powerconserving cancellation of coupling terms by active control. No external force measurements have to be performed. We show asymptotic stability of the generic closed-loop system with an arbitrary number of hierarchy levels. Several simulations confirm our results.
\end{abstract}

\section{INTRODUCTION}

Robots are kinematically redundant if they have more actuated degrees of freedom (DOF) than necessary for a given task. Null space projection techniques [1], [2] can be applied to resolve that redundancy and enable to simultaneously execute additional tasks. Numerically very efficient methods have been developed for hierarchical inverse kinematics [3] and dynamics [4], [5]. In this context, the operational space formulation (OSF) by Khatib [6] provides a powerful tool for task-oriented control of manipulators in a dynamically consistent way. Natale [7] extended a Cartesian impedance controller by a null space task and showed asymptotic convergence of the null space velocity error. In impedance control, the minimization of a quadratic norm of a low priority error has been treated in [8].

However, a thorough stability analysis for the general case with an arbitrary number of priority levels is extremely difficult and not performed so far due to the complexity of the dynamic systems and the applied hierarchies. But before introducing future service robots to domestic environments, this criterion has to be met. Up to now, that field has been covered quite sparsely [7], [9], [10], and most of the concepts only refer to the kinematic case or a hierarchy with only two levels. The key for stability analyses is the kind of representation of the system dynamics. In [11], Park extended the Jacobian matrix of the primary task to an invertible square matrix. As a result, the primary Cartesian coordinates were augmented by some null space velocities. The dynamically consistent pseudoinverse, known from the OSF, was used. As a result, the inertia matrix of the error dynamics becomes block-diagonal. That can be interpreted as a decoupling of the kinetic energies related to the involved tasks. A formulation based on a dynamically consistent extended Jacobian matrix was also utilized by

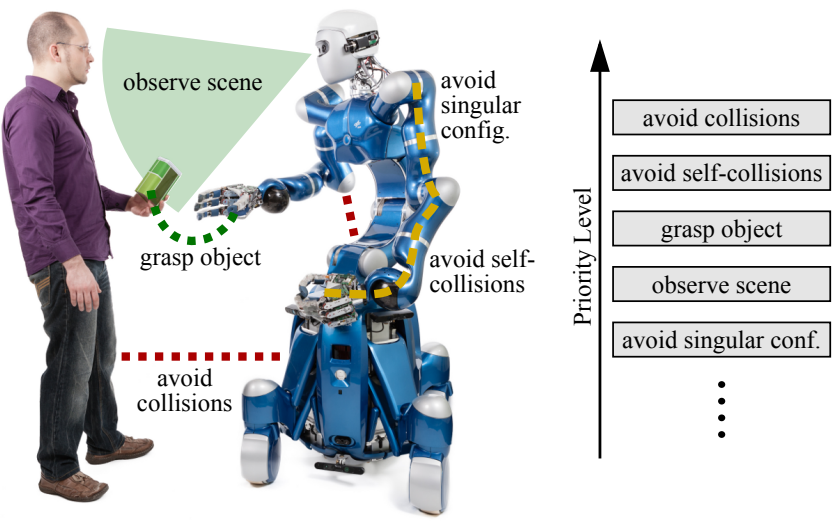

Fig. 1. Example for a task hierarchy with several simultaneous objectives.

Oh [12] for the implementation of an impedance controller. However, the stability analysis was limited to null space damping control. Baillieul [13] described the null space dynamics by introducing additional coordinates. However, new algorithmic singularities may arise, which depend on the particular choice of these coordinates. A decoupling between the tasks can only be achieved by shaping of the reflected inertia. But that requires feedback of the external forces.

The operational space formulation [6] is a well approved method for the implementation of task hierarchies. The applied feedback linearization allows to specify a desired dynamic behavior with particular stability properties. However, the performed inertia shaping, i. e. the realization of a desired inertial behavior, demands feedback of the external forces. If multi-DOF manipulators in dynamic and unstructured environments are considered, for example service robots, contacts are very probable. But even if a robot is equipped with force/torque sensors at the end-effectors, the identification of external forces and torques applied on other parts of the robot structure (e. g. in case of contacts) is not implicated. One way could be to attach a large number of sensors. However, the costs, increasing complexity, energy supply, available space and so on are strong reasons against this solution. Another way would be to use joint torque sensing to compute the external loads. However, a disturbance observer is required, which introduces a lag in turn. Moreover, the accuracy of the dynamic model in the observer has substantial influence on the quality of the measured forces/torques. Summarized, identifying the external loads is a very challenging issue and sometimes even not possible at all [8]. 
In this paper, we extend the work initiated in [9], where the dynamic equations were formulated using specific null space velocities, and a stability analysis was performed. However, only two piority levels were considered. Here we derive the general case of a hierarchy with an arbitrary number of priority levels (Fig. 1) and conclude with a thorough stability proof for the complete system. This work has two main contributions. The first one is the design choice of a particular set of local null space velocities. By enforcing orthogonality of all involved tasks similar to classical null space-based approaches, the order of priority is strictly ensured. The new coordinates combined with a controller, which cancels couplings in the system equations, lead to a largely decoupled dynamics formulation. A key property of the approach is the deliberate avoidance of external force measurements for the feedback control law. The determination of external forces/torques is very challenging and sometimes even impossible. The second main contribution is a thorough stability analysis of the closedloop dynamics. The stability proof guarantees successive, asymptotic stability of all tasks. This is the first proof of that kind for a generic robot with an arbitrary number of DOF and a hierarchy with an arbitrary number of priority levels. Finally, simulations validate the main results of this work including the decoupling, the controller design, and the successive asymptotic convergence of the tasks.

The paper is structured as follows: After the problem formulation in Sec. II, the new dynamics representation is derived in Sec. III. Section IV presents the controller including task execution and power-conserving feedback to cancel velocity dependent couplings between the levels. The stability analysis is conducted in Sec. V and discussed in Sec. VI. Finally, Sec. VII provides several simulations that validate our main contributions, i. e. the decoupling via the new dynamics representation, the controller performance, and the stability proof.

\section{Problem FORMUlation}

The dynamics of an $n$ DOF robot can be formulated as

$$
\boldsymbol{M}(\boldsymbol{q}) \ddot{\boldsymbol{q}}+\boldsymbol{C}(\boldsymbol{q}, \dot{\boldsymbol{q}}) \dot{\boldsymbol{q}}+\boldsymbol{g}(\boldsymbol{q})=\boldsymbol{\tau}+\boldsymbol{\tau}^{\mathrm{ext}}
$$

with the joint configuration given by $\boldsymbol{q} \in \mathbb{R}^{n}$. The inertia matrix $\boldsymbol{M}(\boldsymbol{q}) \in \mathbb{R}^{n \times n}$ is symmetric and p.d., and the Coriolis/centrifugal matrix $\boldsymbol{C}(\boldsymbol{q}, \dot{\boldsymbol{q}}) \in \mathbb{R}^{n}$ complies with $\dot{\boldsymbol{M}}(\boldsymbol{q})=\boldsymbol{C}(\boldsymbol{q}, \dot{\boldsymbol{q}})+\boldsymbol{C}(\boldsymbol{q}, \dot{\boldsymbol{q}})^{T}$. That relation is equivalent to the skew symmetry of $\dot{\boldsymbol{M}}(\boldsymbol{q})-2 \boldsymbol{C}(\boldsymbol{q}, \dot{\boldsymbol{q}})$. One can interpret that property as passivity of (1) with respect to input $\tau$ and output $\dot{\boldsymbol{q}}$. The gravity torques are represented by $\boldsymbol{g}(\boldsymbol{q}) \in \mathbb{R}^{n}$, the generalized forces $\tau \in \mathbb{R}^{n}$ describe the control inputs, and $\tau^{\text {ext }} \in \mathbb{R}^{n}$ are the external forces.

The task hierarchy is supposed to contain $r \in \mathbb{N}$ levels. Thus we introduce $r$ task coordinate vectors by the mappings $\boldsymbol{x}_{i}=\boldsymbol{f}_{i}(\boldsymbol{q}) \in \mathbb{R}^{m_{i}} \forall 1 \leq i \leq r$, with the task dimensions $m_{i} \in \mathbb{N}$. In the order of priority $i=1$ is at the top, and an increasing index stands for a less important task. The mappings from joint to task velocities are defined by the
Jacobian matrices $\boldsymbol{J}_{i}(\boldsymbol{q}) \in \mathbb{R}^{m_{i} \times n} \forall 1 \leq i \leq r$ :

$$
\dot{\boldsymbol{x}}_{i}=\boldsymbol{J}_{i}(\boldsymbol{q}) \dot{\boldsymbol{q}}, \quad \boldsymbol{J}_{i}(\boldsymbol{q})=\frac{\partial \boldsymbol{f}_{i}(\boldsymbol{q})}{\partial \boldsymbol{q}} .
$$

The following analysis is based on the assumption of nonsingular Jacobian matrices, i.e. they are of full row-rank. That restriction will be particularized at the end of Sec. V. The primary task is of dimension $m_{1}<n$ so that a kinematic redundancy of $n-m_{1}$ DOF remains to execute the subtasks in its null space. Moreover, the task hierarchy shall have the following features:

1) A task with lower priority may not disturb any task with higher priority. Hence, a low priority task is executed in the null space of all higher priority tasks.

2) Every task can be described by a p.d. potential function $V_{i}\left(\tilde{\boldsymbol{x}}_{i}(\boldsymbol{q})\right)$ related to the task coordinate $\tilde{\boldsymbol{x}}_{i}=\boldsymbol{x}_{i}-$ $\boldsymbol{x}_{i, d}$, where $\boldsymbol{x}_{i, d}$ is the virtual equilibrium. Damping is injected by the p. d. damping matrix $\boldsymbol{D}_{i}(\boldsymbol{q}) \in \mathbb{R}^{m_{i} \times m_{i}}$.

3) The lowest level in the hierarchy $r$ may be of a larger dimension such that $\sum_{i=1}^{r} m_{i}>n$, e.g. it may be defined in the configuration space.

\section{HierarchicAl Null Space DyNAmics}

In the control design, the dynamics of each priority level will be largely decoupled from all other levels. While the inertial terms can be decoupled by a smart change of the coordinates, the off-diagonal entries in $\boldsymbol{C}(\boldsymbol{q}, \dot{\boldsymbol{q}})$ have to be cancelled by active control (Sec. IV). The following coordinate change is motivated by [9] but it extends the existing work by generalizing to a hierarchy with an arbitrary number of priority levels.

\section{A. Definition of Local Null Space Velocities}

We will reformulate the dynamics (1) to distinguish the different priority levels. In [13], Baillieul introduced additional task coordinates. However, that choice leads to new algorithmic singularities. Instead we follow the two-level approach by Park [14], wherein $n-m_{1}$ local null space velocities $\boldsymbol{v}_{2}=\overline{\boldsymbol{J}}_{2}(\boldsymbol{q}) \dot{\boldsymbol{q}}$ were introduced. Here, we extend that concept to the general case

$$
\boldsymbol{v}_{i}=\overline{\boldsymbol{J}}_{i}(\boldsymbol{q}) \dot{\boldsymbol{q}} \quad \forall 1 \leq i \leq r .
$$

From now on, the dependencies on the system states are omitted in the notations in most of the equations for the sake of simplicity. All mappings $\overline{\boldsymbol{J}}_{i} \in \mathbb{R}^{m_{i} \times n}$ have to be chosen in a way such that the so-called extended Jacobian matrix $\overline{\boldsymbol{J}} \in \mathbb{R}^{n \times n}$ becomes non-singular.

$$
\left(\begin{array}{c}
\boldsymbol{v}_{1} \\
\vdots \\
\boldsymbol{v}_{r}
\end{array}\right)=\overline{\boldsymbol{J}} \dot{\boldsymbol{q}}=\left(\begin{array}{c}
\overline{\boldsymbol{J}}_{1} \\
\vdots \\
\overline{\boldsymbol{J}}_{r}
\end{array}\right) \dot{\boldsymbol{q}} .
$$

For consistent notations, $\boldsymbol{v}_{1}$ and $\overline{\boldsymbol{J}}_{1}$ are used although it is clear that $\boldsymbol{v}_{1}=\dot{\boldsymbol{x}}_{1}$ and $\overline{\boldsymbol{J}}_{1}=\boldsymbol{J}_{1}$ holds. In general the local null space velocities $\boldsymbol{v}_{i} \in \mathbb{R}^{m_{i}}$ are non-integrable. In other words, compatible global null space coordinates $\boldsymbol{n}_{i}(\boldsymbol{q})$ (for $2 \leq i \leq r)$ do not exist such that $\overline{\boldsymbol{J}}_{i}(\boldsymbol{q})=\partial \boldsymbol{n}_{i}(\boldsymbol{q}) / \partial \boldsymbol{q}$ would 
hold. A dynamically consistent null space projection [6], [9] can be applied for $2 \leq i \leq r$ when choosing

$$
\overline{\boldsymbol{J}}_{i}=\left(\boldsymbol{Z}_{i} \boldsymbol{M} \boldsymbol{Z}_{i}^{T}\right)^{-1} \boldsymbol{Z}_{i} \boldsymbol{M},
$$

where $\boldsymbol{Z}_{i}$ is a full row-rank null space base matrix of the augmented Jacobian matrix [2]

$$
\boldsymbol{J}_{i-1}^{\text {aug }}=\left(\begin{array}{c}
\boldsymbol{J}_{1} \\
\boldsymbol{J}_{2} \\
\vdots \\
\boldsymbol{J}_{i-1}
\end{array}\right)
$$

that takes all tasks "down" to level $i-1$ into account. ${ }^{1}$ As a result, the orthogonality of all tasks is enforced so that a strict order of priority is guaranteed. The formal description of that condition is

$$
\boldsymbol{J}_{i-1}^{\text {aug }} \boldsymbol{Z}_{i}^{T}=\mathbf{0} .
$$

However, the question of the choice of $\boldsymbol{Z}_{i}$ for all null space levels is still open.

\section{B. Choice of $\boldsymbol{Z}_{i}$ : Connection to the Classical Approach}

The dynamics are formulated in the coordinates introduced in (4), but they still have to equal the classical approach with the well-known null space projector [6]

$$
\begin{aligned}
\boldsymbol{N}_{i} & =\boldsymbol{I}-\left(\boldsymbol{J}_{i-1}^{\text {aug }}\right)^{T}\left(\left(\boldsymbol{J}_{i-1}^{\text {aug }}\right)^{\boldsymbol{M}+}\right)^{T} \\
& =\boldsymbol{M} \boldsymbol{V}_{i-1}\left(\boldsymbol{V}_{i-1}^{T} \boldsymbol{M} \boldsymbol{V}_{i-1}\right)^{-1} \boldsymbol{V}_{i-1}^{T} .
\end{aligned}
$$

The matrix $\boldsymbol{V}_{i-1} \in \mathbb{R}^{n \times\left(n-\sum_{j=1}^{i-1} m_{j}\right)}$ comprises orthonormal vectors and spans the null space of $\boldsymbol{J}_{i-1}^{\text {aug }}$. The singular value decomposition of $\boldsymbol{J}_{i-1}^{\text {aug }}$ is one way to obtain $\boldsymbol{V}_{i-1}$. A task force from the $i$-th level (with $i \geq 2$ ) is projected via $\boldsymbol{N}_{i} \boldsymbol{J}_{i}^{T}$. In our notations that equals the use of $\overline{\boldsymbol{J}}_{i}^{T} \boldsymbol{Z}_{i} \boldsymbol{J}_{i}^{T}$ instead. In other words, a task force from the original space (2) has to be successively projected three times:

1) Projection into the configuration space via $\boldsymbol{J}_{i}^{T}$.

2) Projection into the new, local directions via $Z_{i}$.

3) Projection back into the configuration space via $\overline{\boldsymbol{J}}_{i}^{T}$. So the solution of

$$
\overline{\boldsymbol{J}}_{i}^{T} \boldsymbol{Z}_{i} \boldsymbol{J}_{i}^{T}=\boldsymbol{N}_{i} \boldsymbol{J}_{i}^{T}
$$

for the matrix $\boldsymbol{Z}_{i}$ has to be found, which is

$$
\begin{aligned}
\boldsymbol{Z}_{i} & =\boldsymbol{J}_{i} \boldsymbol{M}^{-1} \boldsymbol{N}_{i} \\
& =\boldsymbol{J}_{i} \boldsymbol{V}_{i-1}\left(\boldsymbol{V}_{i-1}^{T} \boldsymbol{M} \boldsymbol{V}_{i-1}\right)^{-1} \boldsymbol{V}_{i-1}^{T}
\end{aligned}
$$

for all null space levels but the lowest one $(2 \leq i<r)$. For $i=r$, a projection into $\boldsymbol{J}_{r}$ is useless, because no lower priority levels exist. In other words, the complete remaining null space can be utilized by choosing $\boldsymbol{Z}_{r}=\boldsymbol{V}_{r-1}^{T}$, as also done in [9] for level 2. In fact, the shape of (12) is not surprising: The appearance of $\boldsymbol{J}_{i}$ is justified by the necessity of reducing the available null space of dimension $n \times(n-$ $\sum_{j=1}^{i-1} m_{j}$ ) by projecting it on the subspace that is really used

\footnotetext{
${ }^{1}$ The matrix $\boldsymbol{J}_{i-1}^{\text {aug }}$ is also assumed to have full row-rank. That restriction is discussed at the end of Sec. V.
}

on level $i$. And the remaining terms on the right of (12) have the typical form known from the classical approach (9).

The choice (12) leads to the simple form of

$$
\overline{\boldsymbol{J}}^{-1}=\left(\boldsymbol{J}_{1}^{M+}, \boldsymbol{Z}_{2}^{T}, \ldots, \boldsymbol{Z}_{r}^{T}\right)
$$

for the inverse of the local mapping. The superscript $M+$ denotes the weighted pseudoinverse w.r.t. $M$, i. e. the dynamically consistent one [6]. Replacing (13) in (4) delivers the joint velocities

$$
\dot{\boldsymbol{q}}=\boldsymbol{J}_{1}^{\boldsymbol{M}+} \boldsymbol{v}_{1}+\sum_{i=2}^{r} \boldsymbol{Z}_{i}^{T} \boldsymbol{v}_{i}
$$

incorporating of all task contributions.

\section{Dynamics Formulation}

The above results finally lead to the dynamics

$$
\boldsymbol{\Lambda} \dot{\boldsymbol{v}}+\boldsymbol{\mu} \boldsymbol{v}=\overline{\boldsymbol{J}}^{-T}\left(-\boldsymbol{g}+\boldsymbol{\tau}+\boldsymbol{\tau}^{\mathrm{ext}}\right)
$$

in the new coordinates, where $\boldsymbol{v}=\left(\boldsymbol{v}_{1}^{T}, \ldots, \boldsymbol{v}_{r}^{T}\right)^{T}$. The block-diagonal inertia matrix $\Lambda$ and the (fully coupled) Coriolis/centrifugal matrix $\boldsymbol{\mu}$ are given by

$$
\begin{aligned}
\boldsymbol{\Lambda} & =\overline{\boldsymbol{J}}^{-T} \boldsymbol{M} \overline{\boldsymbol{J}}^{-1}=\operatorname{diag}\left(\boldsymbol{\Lambda}_{1}, \ldots, \boldsymbol{\Lambda}_{r}\right), \\
\boldsymbol{\mu} & =\boldsymbol{\Lambda}\left(\overline{\boldsymbol{J}} \boldsymbol{M}^{-1} \boldsymbol{C}-\dot{\overline{\boldsymbol{J}}}\right) \overline{\boldsymbol{J}}^{-1}, \\
\boldsymbol{\Lambda}_{i} & =\left\{\begin{array}{ll}
\left(\boldsymbol{J}_{1} \boldsymbol{M}^{-1} \boldsymbol{J}_{1}^{T}\right)^{-1} & \text { if } i=1 \\
\boldsymbol{Z}_{i} \boldsymbol{M} \boldsymbol{Z}_{i}^{T} & \text { if } 2 \leq i \leq r
\end{array} .\right.
\end{aligned}
$$

Notice that the block-diagonal shape of $\boldsymbol{\Lambda}$ is a result of choosing $M$ as the weighting for the pseudoinversion in (5) and (13). This specific dynamics formulation using inertiadecoupled null space velocity coordinates is useful for the design of feedback controllers. While such a decoupling could also be achieved via active control by means of feedback linearization (as done in the OSF [6], [15], [16]), the controller design in Sec. IV will aim at a passivitybased compliance controller which deliberately avoids inertia shaping $^{2}$ so that it can be implemented without measurement of external forces acting on the robot.

\section{Control Design}

The control law

$$
\boldsymbol{\tau}=\sum_{i=1}^{r} \boldsymbol{\tau}_{i}+\boldsymbol{\tau}_{\mu}+\boldsymbol{g}
$$

consists of three parts. The first term is composed of the task contributions from all hierarchy levels. The gravity torques are taken into account by $\boldsymbol{g}$, and $\boldsymbol{\tau}_{\mu}$ is a compensation term for off-diagonal entries in the Coriolis/centrifugal matrix as mentioned in Sec. III.

\footnotetext{
${ }^{2}$ Inertia shaping describes the feedback of the actual acceleration to directedly modify the effective, reflected inertia. For that reason, a control without that feedback is often called compliance control.
} 


\section{A. Main Task Control}

Since the main task $(i=1)$ is not constrained by any null space projections, it is treated separately here.

$$
\boldsymbol{\tau}_{1}=-\boldsymbol{J}_{1}^{T}\left(\left(\frac{\partial V_{1}\left(\tilde{\boldsymbol{x}}_{1}\right)}{\partial \boldsymbol{x}_{1}}\right)^{T}+\boldsymbol{D}_{1} \dot{\boldsymbol{x}}_{1}\right)
$$

The damping matrix $\boldsymbol{D}_{1}$ may be configuration dependent as long as it stays positive definite. Inertia shaping is deliberately avoided here because that would require measuring and feedback of the external forces $\tau^{\text {ext }}$.

\section{B. Null Space Control}

The null space task torques $(2 \leq i \leq r)$ have to pass their respective null space projectors (10) additionally:

$$
\boldsymbol{\tau}_{i}=-\overline{\boldsymbol{J}}_{i}^{T} \boldsymbol{Z}_{i} \boldsymbol{J}_{i}^{T}\left(\left(\frac{\partial V_{i}\left(\tilde{\boldsymbol{x}}_{i}\right)}{\partial \boldsymbol{x}_{i}}\right)^{T}+\boldsymbol{D}_{i} \dot{\boldsymbol{x}}_{i}\right) .
$$

Again, the damping matrix $\boldsymbol{D}_{i}$ may be configuration dependent as long as it stays positive definite. The stability properties of this intuitive control law alone are not trivial. As a result of the null space projection via $\overline{\boldsymbol{J}}_{i}^{T} \boldsymbol{Z}_{i}$ or $\boldsymbol{N}_{i}$ [6], respectively, the projected control torques do not represent a passive feedback action in general.

\section{Power-Conserving Cancellation of Coriolis and Centrifu- gal Couplings}

While the inertia matrix $\boldsymbol{\Lambda}$ is already block-diagonal in (15), the projected Coriolis and centrifugal matrix $\boldsymbol{\mu}$ is still fully occupied. To obtain decoupled dynamics, we compensate for the off-(block)diagonal entries in $\boldsymbol{\mu}$. This can be achieved by feedback of the form

$$
\boldsymbol{\tau}_{\mu}=\sum_{i=1}^{r}\left(\overline{\boldsymbol{J}}_{i}^{T}\left(\sum_{j=1}^{i-1} \boldsymbol{\mu}_{i, j} \boldsymbol{v}_{j}+\sum_{j=i+1}^{r} \boldsymbol{\mu}_{i, j} \boldsymbol{v}_{j}\right)\right) .
$$

A block entry in $\boldsymbol{\mu}$ which is located in row $i$ and column $j$ is denoted $\boldsymbol{\mu}_{i, j} \in \mathbb{R}^{m_{i} \times m_{j}}$. An important property of (22) is that it is a power-conserving feedback, i.e. the transmitted power $P_{\mu}=\boldsymbol{\tau}_{\mu}^{T} \dot{\boldsymbol{q}}$ is always zero. This is due to $\boldsymbol{\mu}_{i, j}=$ $-\boldsymbol{\mu}_{j, i}^{T}$, which in turn is a result of the symmetry of $\dot{\boldsymbol{\Lambda}}=\boldsymbol{\mu}+$ $\boldsymbol{\mu}^{T}$ or $\dot{\boldsymbol{M}}=\boldsymbol{C}+\boldsymbol{C}^{T}$, respectively. This feature is very useful from a robustness point of view, because it is independent of parameter uncertainties in the model. While in practice these dynamic effects are of minor importance, their cancellation is essential for the stability analysis in Sec. V in order to obtain largely decoupled dynamics.

\section{Decoupled Closed-Loop Dynamics}

The effect of external forces/torques on the levels is also relevant for the compliance control goal although those loads are not used in the feedback control law. Since the extended Jacobian matrix $\overline{\boldsymbol{J}}$ is invertible, we can replace the external torque $\boldsymbol{\tau}^{\text {ext }}$ uniquely by its components $\boldsymbol{F}_{i}^{\text {ext }} \in \mathbb{R}^{m_{i}}$ related to the corresponding priority level such that the following relation holds:

$$
\boldsymbol{\tau}^{\mathrm{ext}}=\overline{\boldsymbol{J}}^{T}\left(\begin{array}{c}
\boldsymbol{F}_{1}^{\mathrm{ext}} \\
\vdots \\
\boldsymbol{F}_{r}^{\mathrm{ext}}
\end{array}\right)
$$

Applying (19) finally yields the dynamics of the closed loop. On level 1 we obtain

$$
\boldsymbol{\Lambda}_{1} \dot{\boldsymbol{v}}_{1}+\left(\boldsymbol{\mu}_{1,1}+\boldsymbol{D}_{1}\right) \boldsymbol{v}_{1}+\left(\frac{\partial V_{1}\left(\tilde{\boldsymbol{x}}_{1}\right)}{\partial \boldsymbol{x}_{1}}\right)^{T}=\boldsymbol{F}_{1}^{\mathrm{ext}} .
$$

And the lower levels $(2 \leq i \leq r)$ deliver dynamics following

$\boldsymbol{\Lambda}_{i} \dot{\boldsymbol{v}}_{i}+\boldsymbol{\mu}_{i, i} \boldsymbol{v}_{i}+\boldsymbol{Z}_{i} \boldsymbol{J}_{i}^{T}\left(\left(\frac{\partial V_{i}\left(\tilde{\boldsymbol{x}}_{i}\right)}{\partial \boldsymbol{x}_{i}}\right)^{T}+\boldsymbol{D}_{i} \dot{\boldsymbol{x}}_{i}\right)=\boldsymbol{F}_{i}^{\mathrm{ext}}$.

The system (24)-(25) indicates a large decoupling of the priority levels so that the subtasks can be executed in the specified order. One has to keep in mind that an indirect coupling between the levels exists due to the dependencies on $\boldsymbol{q}$, e.g. in the reflected inertias $\boldsymbol{\Lambda}_{i}(\boldsymbol{q})$, as well as due to the occurrence of $\boldsymbol{q}, \dot{\boldsymbol{q}}$ in the remaining Coriolis/centrifugal terms $\boldsymbol{\mu}_{i, i}(\boldsymbol{q}, \dot{\boldsymbol{q}})$. Despite these couplings, the change of the kinetic energy associated with each priority level only depends on the dynamics on the same level. That is a direct consequence of the power-conserving feedback (22).

The decoupled dynamics (24)-(25) represent one of the major contributions of this paper, and they constitute the basis for conducting the following stability analysis.

\section{Stability Analysis}

This stability analysis utilizes the same theorems as [9] but it is an extension to the general case with an arbitrary number of priority levels.

Proposition 1: Consider the system (14), (15) with the control law (19) with (20), (21), and (22). The potential functions $V_{i}\left(\tilde{\boldsymbol{x}}_{i}\right)$ for $i=1 \ldots r$ are positive definite with respect to $\tilde{\boldsymbol{x}}_{i}$, and the damping matrices $\boldsymbol{D}_{i}$ for $i=1 \ldots r$ are symmetric and positive definite. Then the closed-loop system is strictly output passive with respect to the input $\boldsymbol{F}_{1}^{\mathrm{ext}}$ and the output $\dot{\boldsymbol{x}}_{1}$. Suppose also that the Jacobian matrices $\boldsymbol{J}_{i}$ for $i=1 \ldots r$ are of full row-rank in the considered workspace, and $\overline{\boldsymbol{J}}$ is non-singular. The virtual equilibrium configuration $\boldsymbol{q}_{d}$, defined as the unique minimum of all $V_{i}$, is asymptotically stable for the case of free motion, i.e. $\boldsymbol{\tau}^{\mathrm{ext}}=\mathbf{0}$.

Proof: The passivity statement can be easily proven by considering the positive semi-definite function

$$
S_{1}=\frac{1}{2} \boldsymbol{v}_{1}^{T} \boldsymbol{\Lambda}_{1} \boldsymbol{v}_{1}+V_{1}\left(\tilde{\boldsymbol{x}}_{1}\right)
$$

as a storage function for the first priority level. Using the property $\dot{\boldsymbol{\Lambda}}_{1}=\boldsymbol{\mu}_{1,1}+\boldsymbol{\mu}_{1,1}^{T}$ one can show that the time derivative of $S_{1}$ along a solution of the closed-loop system is given by

$$
\dot{S}_{1}=\boldsymbol{v}_{1}^{T} \boldsymbol{F}_{1}^{\mathrm{ext}}-\boldsymbol{v}_{1}^{T} \boldsymbol{D}_{1} \boldsymbol{v}_{1}
$$


from which the passivity property of the Proposition can be directly concluded.

The proof of asymptotic stability will be based on the following theorem which uses the notation of conditional stability [17]

Theorem 1: [17] Let the system

$$
\begin{aligned}
& \dot{\boldsymbol{z}}=\boldsymbol{g}_{1}(\boldsymbol{z})+\boldsymbol{g}_{2}(\boldsymbol{z}) \boldsymbol{u}, \\
& \boldsymbol{y}=\boldsymbol{h}(\boldsymbol{z})
\end{aligned}
$$

with state $\boldsymbol{z} \in \mathbb{R}^{n}$, input $\boldsymbol{u} \in \mathbb{R}^{m}$, and output $\boldsymbol{y} \in \mathbb{R}^{m}$ be strictly output passive for the output $\boldsymbol{y}=\boldsymbol{h}(\boldsymbol{z})$. Let further $\mathcal{A}$ be the largest positively invariant set contained in $\left\{\boldsymbol{z} \in \mathbb{R}^{n} \mid \boldsymbol{h}(\boldsymbol{z})=\mathbf{0}\right\}$. If the equilibrium $\boldsymbol{z}^{*}$ is asymptotically stable conditionally to $\mathcal{A}$, then it is asymptotically stable for $\boldsymbol{u}=\mathbf{0}$.

By iteratively applying Theorem 1, one can show the stability statement in Proposition 1. We have already proven strict output passivity w. r. t. output $\dot{\boldsymbol{x}}_{1}$. From (24) we can conclude that, for $\boldsymbol{F}_{1}^{\text {ext }}=\mathbf{0}$ (free motion), the largest positively invariant set contained in $\left(\boldsymbol{q}, \boldsymbol{v}_{1}=\mathbf{0}, \boldsymbol{v}_{2}, \ldots, \boldsymbol{v}_{r}\right)$ is

$$
\mathcal{A}_{1}=\left\{\left(\boldsymbol{q}, \boldsymbol{v}_{1}=\mathbf{0}, \boldsymbol{v}_{2}, \ldots, \boldsymbol{v}_{r}\right) \mid \boldsymbol{f}_{1}(\boldsymbol{q})=\boldsymbol{x}_{1, d}\right\} .
$$

Therefore, we have to show asymptotic stability of the system conditionally to the set $\mathcal{A}_{1}$. In order to achieve that, we must use Theorem 1 again. But now, it is applied to the system conditionally to $\mathcal{A}_{1}$. We consider the storage function

$$
S_{i}=\frac{1}{2} \boldsymbol{v}_{i}^{T} \boldsymbol{\Lambda}_{i} \boldsymbol{v}_{i}+V_{i}\left(\tilde{\boldsymbol{x}}_{i}\right)
$$

for the priority level $i=2$. The time derivative of $S_{2}$ along the solutions of (24), conditionally to $\mathcal{A}_{1}$, is given by

$$
\begin{aligned}
\dot{S}_{2}= & \boldsymbol{v}_{2}^{T} \boldsymbol{F}_{2}^{\mathrm{ext}}-\boldsymbol{v}_{2}^{T} \boldsymbol{Z}_{2} \boldsymbol{J}_{2}^{T} \boldsymbol{D}_{2} \frac{\partial \boldsymbol{x}_{2}}{\partial \boldsymbol{q}} \dot{\boldsymbol{q}} \\
& -\boldsymbol{v}_{2}^{T} \boldsymbol{Z}_{2} \boldsymbol{J}_{2}^{T}\left(\frac{\partial V_{2}\left(\tilde{\boldsymbol{x}}_{2}\right)}{\partial \boldsymbol{x}_{2}}\right)^{T}+\frac{\partial V_{2}\left(\tilde{\boldsymbol{x}}_{2}\right)}{\partial \boldsymbol{x}_{2}} \frac{\partial \boldsymbol{x}_{2}}{\partial \boldsymbol{q}} \dot{\boldsymbol{q}}
\end{aligned}
$$

From (14) and $\boldsymbol{J}_{i} \boldsymbol{Z}_{j}^{T}=\mathbf{0} \forall j>i$ (which follows from (7)), we can conclude that, in the set $\mathcal{A}_{1}$, the term $\left(\partial \boldsymbol{x}_{2} / \partial \boldsymbol{q}\right) \dot{\boldsymbol{q}}$ simplifies to $\left(\partial \boldsymbol{x}_{2} / \partial \boldsymbol{q}\right) \dot{\boldsymbol{q}}=\left(\partial \boldsymbol{x}_{2} / \partial \boldsymbol{q}\right) \boldsymbol{Z}_{2}^{T} \boldsymbol{v}_{2}$. The last two terms in (29) cancel out and we obtain

$$
\dot{S}_{2}=\boldsymbol{v}_{2}^{T} \boldsymbol{F}_{2}^{\mathrm{ext}} \underbrace{-\boldsymbol{v}_{2}^{T} \boldsymbol{Z}_{2} \boldsymbol{J}_{2}^{T} \boldsymbol{D}_{2} \boldsymbol{J}_{2} \boldsymbol{Z}_{2}^{T} \boldsymbol{v}_{2}}_{\leq 0}
$$

from which we conclude strict output passivity w. r. t. input $\boldsymbol{F}_{2}^{\text {ext }}$ and output $\boldsymbol{v}_{2}$. That passivity property only holds conditionally to the set $\mathcal{A}_{1}$, i. e. after convergence of the main task. It remains to show asymptotic stability of the free system conditionally to the largest positively invariant set in $\left\{\left(\boldsymbol{q}, \boldsymbol{v}_{1}=\mathbf{0}, \boldsymbol{v}_{2}=\mathbf{0}, \boldsymbol{v}_{3}, \ldots, \boldsymbol{v}_{r}\right) \mid \boldsymbol{f}_{1}(\boldsymbol{q})=\boldsymbol{x}_{1, d}\right\}$, which is given by the set

$$
\left.\mathcal{A}_{2}=\mathcal{A}_{1} \bigcap\left\{\boldsymbol{v}_{2}=\mathbf{0}, \boldsymbol{Z}_{2}(\boldsymbol{q})\left(\partial V_{2}\left(\boldsymbol{x}_{2}(\boldsymbol{q})\right)\right) / \partial \boldsymbol{q}\right)^{T}=\mathbf{0}\right\} .
$$

In order to show that, we proceed similarly as before. Starting with (28) for $i=3$, we follow the same steps as above to prove strict output passivity for the output $\boldsymbol{v}_{3}$. The procedure can be iteratively repeated until the lowest priority level $i=r$ is reached. It is important to notice that, if we consider the dynamics of the $i$-th priority level conditionally to the set

$$
\begin{gathered}
\mathcal{A}_{i-1}:=\left\{\left(\boldsymbol{q}, \boldsymbol{v}_{1}=\mathbf{0}, \boldsymbol{v}_{2}, \ldots, \boldsymbol{v}_{r}\right) \mid \boldsymbol{f}_{1}(\boldsymbol{q})=\boldsymbol{x}_{1, d},\right. \\
\left.\boldsymbol{Z}_{j} \boldsymbol{J}_{j}^{T}\left(\frac{\partial V_{j}\left(\tilde{\boldsymbol{x}}_{j}\right)}{\partial \boldsymbol{x}_{j}}\right)^{T}=\mathbf{0}, \boldsymbol{v}_{j}=\mathbf{0}, j=2 \ldots i-1\right\},
\end{gathered}
$$

(where the higher priority tasks have already converged), all velocities $\boldsymbol{v}_{j}$ for $j<i$ are zero. At the lowest priority level $i=r$, we consider (28) as a Lyapunov function candidate. From $\dot{S}_{r}=\boldsymbol{v}_{r}^{T} \boldsymbol{F}_{r}^{\text {ext }}-\boldsymbol{v}_{r}^{T} \boldsymbol{Z}_{r} \boldsymbol{J}_{r}^{T} \boldsymbol{D}_{r} \boldsymbol{J}_{r} \boldsymbol{Z}_{r}^{T} \boldsymbol{v}_{r}$ we can conclude conditional stability of the system conditionally to the set $\mathcal{A}_{r-1}$ for the case of free motion $\boldsymbol{F}^{\mathrm{ext}}=\mathbf{0}$.

Additionally, conditional asymptotic stability of the equilibrium $\left(\boldsymbol{q}=\boldsymbol{q}_{d}, \boldsymbol{v}=\mathbf{0}\right)$ can be ensured by referring to LaSalle's invariance principle. According to that, the state converges to the largest positively invariant set contained in $\mathcal{A}_{r-1}$ where $\boldsymbol{v}_{r}=\mathbf{0}$. By observing the closedloop dynamics (25), one concludes that this set requires $\boldsymbol{Z}_{i} \boldsymbol{J}_{i}^{T}\left(\partial V_{i}\left(\tilde{\boldsymbol{x}}_{i}\right) / \partial \boldsymbol{x}_{i}\right)^{T}=\mathbf{0}$ for all null space levels $i=$ $2 \ldots r$. Since each $\boldsymbol{Z}_{i}$ is a full row-rank null space base matrix, the point $\boldsymbol{q}=\boldsymbol{q}_{d}$ is an isolated point in this set. Thus, the system is asymptotically stable conditionally to the set $\mathcal{A}_{r-1}$. Combining that and the strict output passivity on each hierarchy level, we go back in the iteration and apply Theorem 1 to conclude asymptotic stability conditionally to the sets $\mathcal{A}_{i}$ for $i=r-1 \ldots 1$. The asymptotic stability conditionally to the set $\mathcal{A}_{1}$, together with the passivity property of the main task, lets us conclude asymptotic stability of the complete system for the case of free motion.

Intuitively, the iterative application of Theorem 1 can be interpreted as a sequential convergence of the different tasks according to their priority levels. In addition to asymptotic stability, the proof also contains a hierarchical passivity statement in the sense that after convergence of all higher priority tasks, each lower priority task $i$ shows a passive behavior for the input $\boldsymbol{F}_{i}^{\text {ext }}$ and the respective output $\boldsymbol{v}_{i}$. That passivity property does not necessarily demand that the desired equilibrium configurations are reached on each level, an arbitrary steady state is sufficient.

The whole analysis refers to the case of non-singular Jacobian matrices. A change in the rank of Jacobian matrices is not dealt with in any stability analysis so far. Combining singularity-robust techniques [18], [19] and stability analyses is topic of current research in the robotics community. The condition of non-singular Jacobian matrices is also a restriction on the workspace in which the stability proof is valid, cf. Proposition 1. Note that the classical approaches [6], [2] suffer from the same problems.

\section{Discussion}

In this section, the properties of our approach are summarized and comparisons with other approaches are presented briefly. The analysis performed here ensures asymptotic stability and guarantees successive convergence w. r. t. the order of priority. Approaches based on the OSF [6], [4] provide stronger stability statements (exponential stability). However, in order to achieve that, external forces have to be used in 


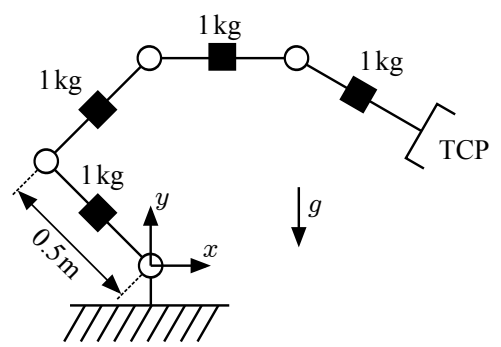

Fig. 2. Sketch of the planar simulation model. The four links are connected via four revolute joints. Each link is modeled by a point mass of $1 \mathrm{~kg}$ which is placed in the middle of a bar with length $0.5 \mathrm{~m}$. The dynamics are simulated using $g=9.81 \mathrm{~m} / \mathrm{s}^{2}$. Zero joint angles are obtained in the upright configuration.

TABLE I

INITIAL CONDITIONS AND DESIRED VALUES

\begin{tabular}{|c|c|c|c|}
\hline Priority & Description & Initial Value & Goal Value \\
\hline \hline \multirow{2}{*}{ Level 1 } & $x$ of TCP & $0.84 \mathrm{~m}$ & $0.90 \mathrm{~m}$ \\
\cline { 2 - 4 } & $y$ of TCP & $0.96 \mathrm{~m}$ & $0.8 \mathrm{~m}$ \\
\hline Level 2 & $\sum_{i=1}^{4} \boldsymbol{q}_{i}$ & $-1.37 \mathrm{rad}$ & $-1.57 \mathrm{rad}$ \\
\hline Level 3 & $\boldsymbol{q}_{1}$ & $0.40 \mathrm{rad}$ & $0.35 \mathrm{rad}$ \\
\hline
\end{tabular}

the control law. As mentioned in the introduction, such a measurement or identification is very difficult, deteriorates the robustness, and is even impossible in some cases. In the approach proposed here, no external force measurements have to be performed, which constitutes the main difference to most of the state-of-the-art methods. In summary, one can say that OSF concepts are better qualified for trajectory tracking (without contacts), and the compliance control in this paper is better suited for interaction tasks.

Another aspect worth mentioning is the computation time. Several numerically costly calculations have to be performed. In inverse kinematics and dynamics, a variety of dedicated solvers have been developed [3], [5]. In order to apply the controller in real-time scenarios, one should consider the usage of such efficient numerical algorithms.

\section{Simulations}

Simulations with a planar 4 DOF robot are performed to confirm the results of Sec. III/IV (decoupled dynamics, controller) and of Sec. V (successive convergence). A schematic representation of the simulated model is given in Fig. 2. In both runs, three priority levels are applied:

Level 1: translational Cartesian compliance of the TCP,

Level 2: compliance of the TCP rotation,

Level 3: joint compliance of first joint.

The regulation case is considered for all hierarchy levels. The initial and goal values are listed in Table I.

\section{A. Simulation \#1 - Validation of the Controller and the Decoupling}

The controller parameterization is provided in Table II. In this experiment the damping is set comparatively high on all levels and the task execution as well as the decoupling quality are analyzed. The errors in the operational space are given in Fig. 3. They all converge to zero within less than $0.5 \mathrm{~s}$.
TABLE II

CONTROLLER PARAMETERIZATION

\begin{tabular}{|c|c|c||c|c|}
\hline Priority & Gain & Unit & Simulation \#1 & Simulation \#2 \\
\hline \hline \multirow{2}{*}{ Level 1 } & $\boldsymbol{K}_{1}$ & $\mathrm{~N} / \mathrm{m}$ & $\operatorname{diag}(1000,1000)$ & $\operatorname{diag}(500,500)$ \\
\cline { 2 - 5 } & $\boldsymbol{D}_{1}$ & $\mathrm{Ns} / \mathrm{m}$ & $\operatorname{diag}(40,40)$ & $\operatorname{diag}(30,30)$ \\
\hline \multirow{2}{*}{ Level 2 } & $\boldsymbol{K}_{2}$ & $\mathrm{Nm} / \mathrm{rad}$ & 800 & 600 \\
\cline { 2 - 5 } & $\boldsymbol{D}_{2}$ & $\mathrm{Nms} / \mathrm{rad}$ & 5 & 1.5 \\
\hline \multirow{2}{*}{ Level 3 } & $\boldsymbol{K}_{3}$ & $\mathrm{Nm} / \mathrm{rad}$ & 2400 & 600 \\
\cline { 2 - 5 } & $\boldsymbol{D}_{3}$ & $\mathrm{Nms} / \mathrm{rad}$ & 15 & 1.5 \\
\hline
\end{tabular}
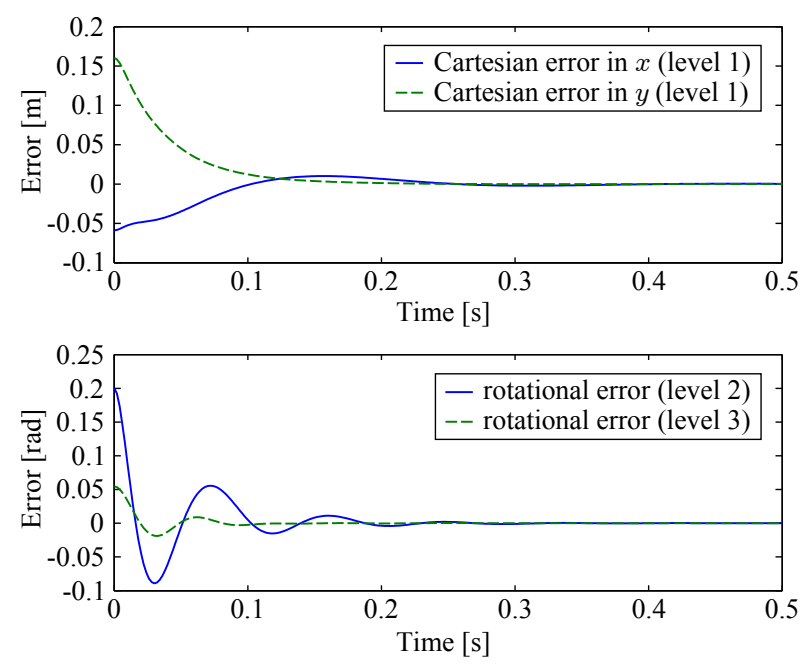

Fig. 3. Simulation \#1: The errors in the operational spaces of the hierarchy levels converge to zero. Initial and goal values are summarized in Table I.

The corresponding control torques from the three levels are depicted in Fig. 4. Solving the dynamics (24)-(25) for the accelerations, one can evaluate the undesired effects induced by active control on the other priority levels, see Fig. 5 . The couplings stay in a range that does not lead to any relevant perturbations. One can conclude that a strict hierarchy is realized, and the conditions imposed in Sec. IV are met. The figure also shows that, in this simulation, the remaining couplings due to the dependencies of the mass matrices and the Coriolis/centrifugal terms on $\boldsymbol{q}, \dot{\boldsymbol{q}}$ can be disregarded.

\section{B. Simulation \#2 - Validation of the Stability Proof}

The controller is parameterized according to Table II and the reference values are taken from Table I again. Now the damping is set very low in order to demonstrate the stability properties without eliminating undesired effects by energy dissipation through damping injection. Fig. 6 shows the errors on all levels of the hierarchy. As expected, the lower damping leads to more distinct oscillations. The main task (top) is undisturbed, and it converges very fast in both Cartesian directions. For $t>0.4 \mathrm{~s}$, the primary task error is almost zero, and it is not affected by the remaining null space motions (bottom) as stated in Sec. V. The latter require a longer time to get into a steady state at zero. The energies are depicted in Fig. 7. In accordance to the primary task error, the total energy on the first level converges to zero. The two bottom diagrams illustrate the energy contributions on the two null space levels. When looking at the interac- 

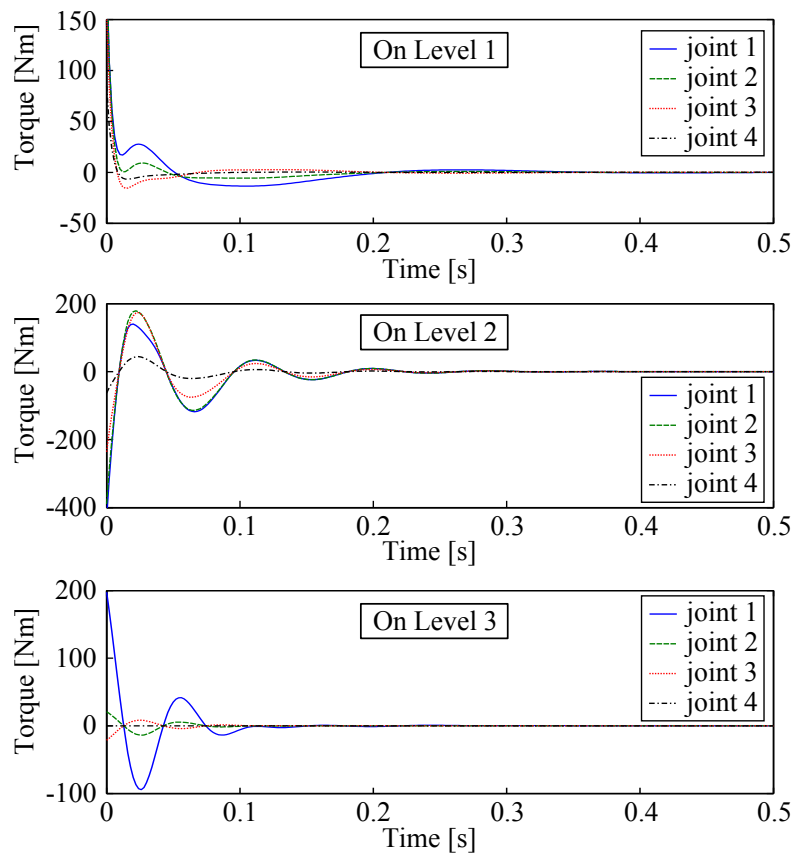

Fig. 4. Simulation \#1: Contributions of the control input depending on the corresponding hierarchy level. All signals lie in about the same range, but they do not interfere with each other (w. r. t. the operational space behavior).

tion between potential and kinetic energy, the low damping with regard to the eigenfrequencies becomes obvious here, particularly on level 3. Obviously, the total energies on level 2 and level 3 are not monotonically decreasing. However, that complies with the stability proof based on conditional stability. If one considers the fact that the total energy of the high priority task (level 1) requires at least $0.25 \mathrm{~s}$ to reach about zero, the behavior on level 2 is proper. For $t>0.25 \mathrm{~s}$, the total energy on level 2 monotonically decreases. The same applies to the third level w.r.t. the second level for $t>0.4 \mathrm{~s}$. The stability analysis demands a monotonically decreasing total energy on level 3 as soon as level 2 has converged.

\section{SUMMARY}

This paper has two main contributions: First, we represented the classical concept of hierarchy-based control for torque-controlled, redundant manipulators in a new formulation of the dynamic equations. These constituted a large, hierarchical decoupling. Second, we performed a thorough stability analysis with a strict task hierarchy realized by means of null space projections. Asymptotic stability and successive convergence of all hierarchy levels were concluded. Among others, that was made possible by adapting standard control algorithms through power-conserving feedback of dynamic couplings between the different priority levels. Although hierarchical control approaches based on null space projections have been abundantly used in the last decades, such a stability analysis has not been conducted so far. The major difference of our concept compared to the classical operational space approach is that no measurements of external forces/torques have to be performed. Especially
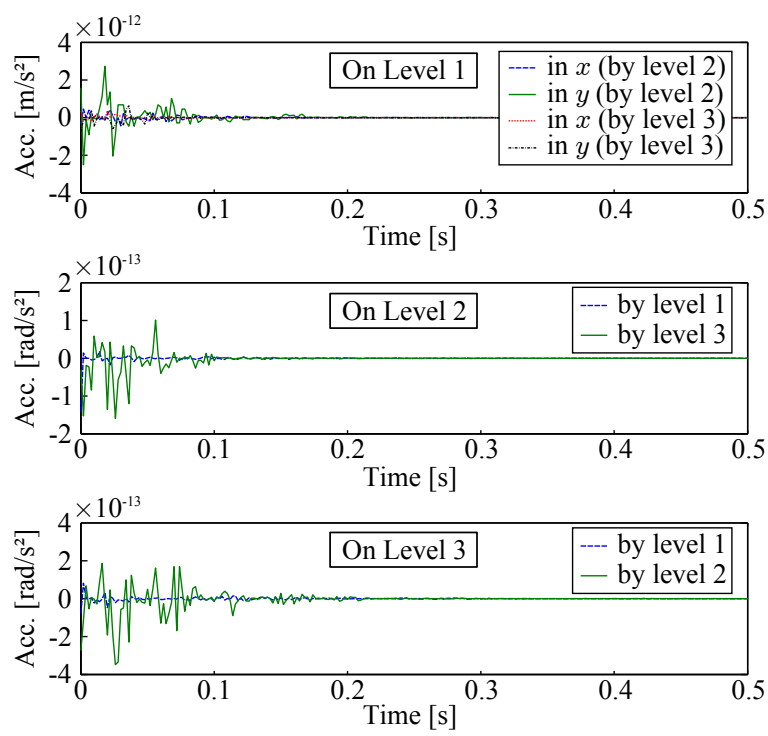

Fig. 5. Simulation \#1: The plots depict disturbing accelerations on the levels induced by the control inputs from the other levels. The values stay in a range that is negligible. In conclusion, a strict hierarchy is realized.
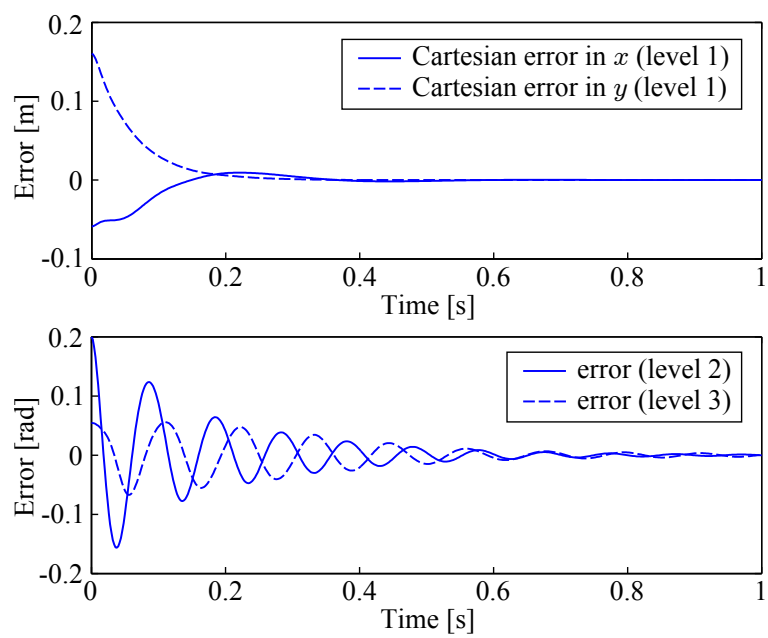

Fig. 6. Simulation \#2: Errors on the three priority levels. The primary task (upper plots) converges very fast, while the null space tasks (bottom plots) require a longer time to get into a steady state. The damping in the null space is set very low compared to the primary Cartesian task.

when considering relevant scenarios for redundant robots such as human-robot interaction or multiple contacts, the exact measurement or identification of external loads is a very challenging task and sometimes not possible at all. Simulations on a 4 DOF system validated the main results of our approach.

\section{REFERENCES}

[1] Y. Nakamura, H. Hanafusa, and T. Yoshikawa, "Task-Priority Based Redundancy Control of Robot Manipulators," International Journal of Robotics Research, vol. 6, no. 2, pp. 3-15, June 1987.

[2] B. Siciliano and J.-J. Slotine, "A General Framework for Managing Multiple Tasks in Highly Redundant Robotic Systems," in Proc. of the 5th International Conference on Advanced Robotics, June 1991, pp. 1211-1216. 

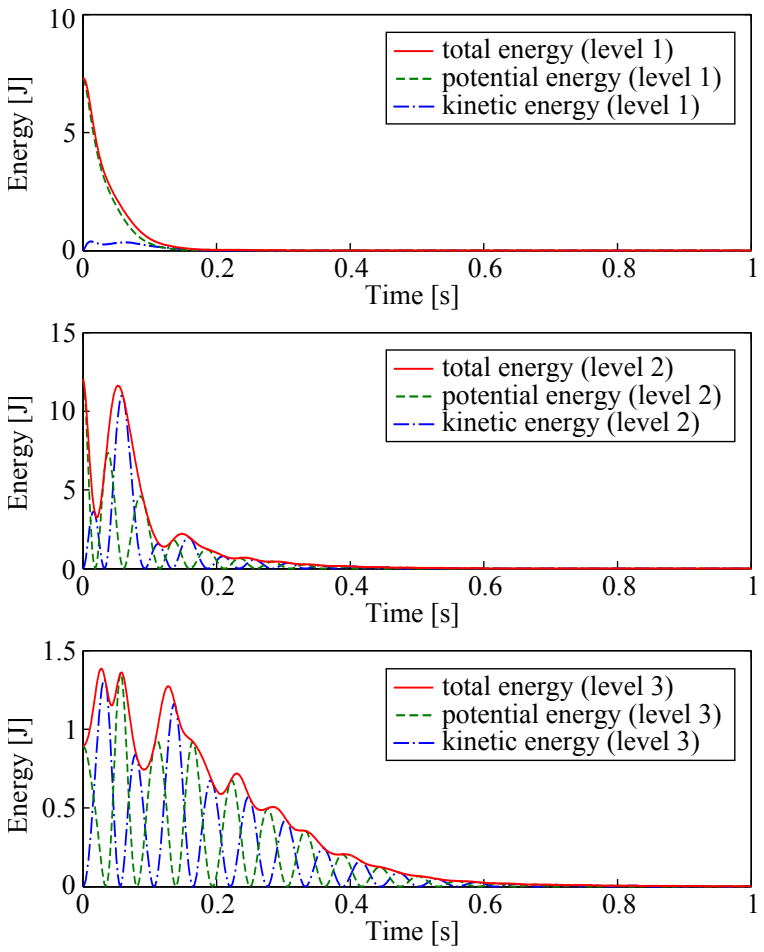

Fig. 7. Simulation \#2: Energies on the three priority levels. The main task (level 1) is decoupled from the null space behavior and converges fast. The total energy on a null space level is only monotonically decreasing after all higher priority tasks have already converged.

[3] A. Escande, N. Mansard, and P.-B. Wieber, "Fast Resolution of Hierarchized Inverse Kinematics with Inequality Constraints," in Proc. of the 2010 IEEE International Conference on Robotics and Automation, May 2010, pp. 3733-3738.

[4] N. Mansard, O. Khatib, and A. Kheddar, "A Unified Approach to Integrate Unilateral Constraints in the Stack of Tasks," IEEE Transactions on Robotics, vol. 25, no. 3, pp. 670-685, June 2009.

[5] N. Mansard, "A Dedicated Solver for Fast Operational-Space Inverse Dynamics," in Proc. of the 2012 IEEE International Conference on Robotics and Automation, May 2012, pp. 4943-4949.
[6] O. Khatib, "A Unified Approach for Motion and Force Control of Robot Manipulators: The Operational Space Formulation," IEEE Journal of Robotics and Automation, vol. RA-3, no. 1, pp. 43-53, February 1987.

[7] C. Natale, B. Siciliano, and L. Villani, "Spatial impedance control of redundant manipulators," in IEEE Int. Conf. on Robotics and Automation, 1999, pp. 1788-1793.

[8] R. Platt, M. Abdallah, and C. Wampler, "Multi-Priority Cartesian Impedance Control," in Proc. of Robotics: Science and Systems, 2010.

[9] C. Ott, A. Kugi, and Y. Nakamura, "Resolving the Problem of Non-integrability of Nullspace Velocities for Compliance Control of Redundant Manipulators by using Semi-definite Lyapunov functions," in Proc. of the 2008 IEEE International Conference on Robotics and Automation, May 2008, pp. 1999-2004.

[10] G. Antonelli, "Stability Analysis for Prioritized Closed-Loop Inverse Kinematic Algorithms for Redundant Robotic Systems," IEEE Transactions on Robotics, vol. 25, no. 5, pp. 985-994, October 2009.

[11] J. Park, "Analysis and Control of Kinematically Redundant Manipulators: An Approach Based on Kinematically Decoupled Joint Space Decomposition," Ph.D. dissertation, Pohang University of Science and Technology, 1999.

[12] Y. Oh, W. Chung, and Y. Youm, "Extended impedance control of redundant manipulators based on weighted decomposition of joint space," Journal of Robotic Systems, vol. 15, no. 5, pp. 231-258, 1998.

[13] J. Baillieul, "Kinematic programming alternatives for redundant manipulators," in Proc. of the 1985 IEEE International Conference on Robotics and Automation, March 1985, pp. 722-728.

[14] J. Park, W. Chung, and Y. Youm, "On Dynamical Decoupling of Kinematically Redundant Manipulators," in Proc. of the 1999 IEEE/RSJ International Conference on Intelligent Robots and Systems, October 1999, pp. 1495-1500.

[15] O. Khatib, L. Sentis, J. Park, and J. Warren, "Whole-Body Dynamic Behavior and Control of Human-like Robots," International Journal of Humanoid Robots, vol. 1, no. 1, pp. 29-43, March 2004.

[16] L. Sentis and O. Khatib, "Synthesis of Whole-Body Behaviors through Hierarchical Control of Behavioral Primitives," International Journal of Humanoid Robotics, vol. 2, no. 4, pp. 505-518, January 2005.

[17] A. van der Schaft, $L_{2}$-Gain and Passivity Techniques in Nonlinear Control, 2nd ed. Springer, Berlin Heidelberg, 2000.

[18] N. Mansard, A. Remazeilles, and F. Chaumette, "Continuity of Varying-Feature-Set Control Laws," IEEE Transactions on Automatic Control, vol. 54, no. 11, pp. 2493-2505, November 2009.

[19] A. Dietrich, T. Wimböck, A. Albu-Schäffer, and G. Hirzinger, "Integration of Reactive, Torque-Based Self-Collision Avoidance Into a Task Hierarchy," IEEE Transactions on Robotics, vol. 28, no. 6, pp. 1278-1293, December 2012. 